\title{
A Theoretical Framework of Smart System Modelling of Crop Based on GAN and IoT Platform
}

\author{
Wasim Ahmad Ansari ${ }^{1}$, Prof. Yang Yuwang ${ }^{2}$ \\ \{dr.wasim.scholar@njust.edu.cn ${ }^{1}$ \} \\ School of Computer Science and Engineering, Nanjing University of Science \& Technology, \\ China ${ }^{1}$ \\ School of Computer Science and Engineering, Nanjing University of Science \& Technology, \\ China $^{2}$
}

\begin{abstract}
The advent of the communication and cutting edge technologies is playing a key role in reforming the various sectors and so as in the field of the agronomy. The use of the internet of the things (IOT) technologies is setting the new horizon through automation intelligence for the precision agriculture (PAg) is nothing but combination of communication technologies using information integration along with the control technologies in the field of agriculture. Greenhouses facing the problem of barren land basically need such systems potentially relevant to control re-circulation in the optimal way needed to such green houses. In this paper the proposed system is segregated essentially into three parts to have better understanding of the system architecture. These first, second and parts are termed as Cyber Physical System (CPS) layer, edge computing layer and cloud computing-gan layer respectively with use of IOT protocols like message queuing telemetry transport (MQTT) protocol or constrained application protocol (CoAP) for communication purpose [3]. The third and the final layer which is termed as cloud-gan layer gets further bifurcated into two parts i.e. Cloud part and the GAN [1] part. The cloud platform is responsible to perform the historical and current data analysis and the second part known as generative adversarial nets (GAN) based on the two multilayer perceptron models known as generator and discriminator. This paper describes the theoretically concept of using GAN with the three tier system discussed above. GAN uses the analyzed data from the cloud part and then generator generates the data distribution (probability distribution) and then discriminator discriminate the data on the certain required defined in the system to enable the system automation to make more optimized decision to enhance the system ability to increase the production of the intended crops. GAN part works on the min max concept in which discriminator is trained to maximize the level assignment probability whereas generator is getting trained to minimize the chances of mistakes done by discriminator to support more accurate result. The concept of the GAN with the existing system mentioned above will enable the system to enhance its intelligence in order to make more sharpen and accurate decision..
\end{abstract}

Keywords: Precision Agriculture, IOT, Edge Computing, Cloud Computing, Automation, GAN.

\section{Introduction}

Many organizations and people in the world has put their efforts to strengthen the improvement made in the field of agronomy. In this paper, the proposal of a system based on the three tier with the use of the Generative Adversarial Nets (GAN) concept. The system comprises a local crop side 
installed platform with edge computing plane and the cloud-gan platform in the field of the Precision Agriculture (PAg) which is nothing but in the agriculture term it is the mixture of integrating the information using communication along with the controlled technologies. The use of the appearance of the internet of the things (IOT) technologies and the cutting edge cloud computing technologies further provides the facilities to enhance the system capabilities. In terms of reducing cost oriented scenario and inn order to cover all PAg management efficiently, this flexible platform is proposed to cope with the soilless nature atmosphere uses saline water in moderate way in whole circulation green houses. The state of the art in the up-to date agriculture field leads the advances to the barren culture proliferation [2] termed as hydroponics meant to productivity gain. It is very obvious that vegetable crops concerned to open field required fertilizers much less than that of the hydroponics system but likely causes problems of the land and water eutrophication due to the released drainage discharging around nitrate as $31 \%$ and potassium as $48 \%$ [3].

However, from the above discussion one can easily inferred that these greenhouses require a large amount of water and fertilizers which causes the challenge to cope the management of the same in effective and efficient way to reduce the costs of the modernistic agriculture. These closed hydroponic system is very feasible to the closed field crops production. Such systems are also termed as full re-circulation system. Since these systems are based on the nutrient solution with inbuilt salinity, so if these systems are adopted where the water salinity is high, like Mediterranean areas, may affect the crops productivity due to salinity. Nutrient solution already has electrical conductivity high due to built-in salinity, so it causes a challenge to deal to optimize the electrical conductivity and prevent the release of drainage in order to increase the crops productivity. It can simply be done by adding water with least conductivity using a RO (Reverse Osmosis) system. However, these kind of full re-circulation systems require a lot of information concerning their performance. This is the point where the communication technologies come into the picture to gather the needed information from the crop field to the cloud for analysis purpose in order to maximize the necessary functioning of the system.

The cutting edge technologies such as internet of the things (IoT) and cloud computing have led the fantastic modern development in the efficient monitoring, handling as well as controlled systems in 
the field of agronomy. These systems are sufficiently able to manage the crop status due to the presence of the effective sensors and the available actuators are suffice to handle the affecting climate factors, irrigation required as well as using the required nutrients to strengthen the target agriculture land [4]. In the current scenario, the internet of things (IoT), the technology used for communication abilities along with actuators as well as sensors [5] providing an ameliorated performance platform to the PAg domain [6]. The use of the information integration along with communication technologies employing industrial automation leads to the precision agriculture [7].

The highly effective sensors as well as actuators provide the intended crop's stratum and the management of integration along with the necessary nutrients and climate condition parameters are equipped with powerful automation nodes are nothing but just the gateways of the internet of things (IoT). The evolved technologies in the automation and communication are playing a sophisticated role enabling such systems robust and reliable with firmware to make communication between the highly changeable hardware as required with the use of wireless channels which employ the needed communication technologies in order to optimize the overall costs required in infrastructure, power consumption as well as networking the whole precision agriculture model [8]. Cyber Physical System (CPS) is a term considered scientifically and basically employed the distributed network embedded system in order to collect and communicate data using sensors and actuators for automation purpose. This hardware is employed to hook up with industrial processes, comprising the ability of storing, evaluating as well as communicating [9].

The proposed model includes a local crop platform which has CPS (Cyber Physical System) whose main work is to collect the necessary data from the crop field through installed devices in order to perform atomic tasks in real time. The main PAg tasks such as monitoring and handling are the responsibility of the second platform known as edge platform. These monitoring and handling actions also ensure access network system reliability against its failure. Last but not the least the third platform termed as could-gan platform performs on the historical and current gathered data i.e. the big data with the help of analytics module. As far as the communication is concerned between the CPS and edge plan, this system uses the Message Queuing Telemetry Transfer (MQTT) or Constrained Application Protocol (CoAP). In order to make access to the clouds, Next Generation 
Service Interface (NGSI) is used. This entire system is designed for the greenhouses to enhance the environmental production of the crops.

The GAN (Generative Adversarial Nets), performance increasing component of the proposed system with the theoretical approach, follows the adversarial process comprising two models need to get trained simultaneously. These two models are termed as $\mathrm{G}_{\mathrm{a}}$ : generative model used for the data generation also acts as a data distribution turned in probability distribution and second one is as $\mathrm{D}_{\mathrm{a}}$ : discriminative model used to evaluate the probability whether a sample is from trained data instead of $G_{a}$ i.e. the $G_{a}$ generated distribution. $G_{a}$ is trained to minimize the probability of occurring a mistake done by $\mathrm{D}_{\mathrm{a}}$. Here, two arbitrary functions $\mathrm{G}_{\mathrm{a}}$ and $\mathrm{D}_{\mathrm{a}}$ are used to produce a unique solution

having $\mathrm{G}_{\mathrm{a}}$ rendering the training data distribution and $\mathrm{D}_{\mathrm{a}}$ rendering to $\frac{1}{2}$ elsewhere. The two models are multilayer perceptron models trained with backpropagation. [1].

The state of the art of the internet of the things (IoT), cloud computing along with Generative Adversarial Nets (GAN) will be providing a new horizon in the field of crop model aiming to increase the productivity of the crops in the full re-circulation greenhouses. This model comes with strength does not need Markov chains in training or production of samples.

\section{State of The Art}

The group [10] brought a rule based idea evolving the concept of the control area. Initially precision agriculture (PAg) emphasized actuators automatic control based on the sensor data collected from the crop whereas the group [11] presented the event driven solution. In order to assist farmers in managing and taking decision required, in the last few years precision agriculture (PAg) had played a key role by comprising the integration of the latest information along with communication technologies.

A crop managing system employed remote desktop as well as regular client/server application described by the group [12]. Using actuators along with sensors to make communication to gateway nodes as well as internet, PAg through Internet of the things (IoT) integration has been evolved. 
Since such systems are used at the farming site where cellular/wifi base stations may be available hardly, so the new IoT hardware with the latest capabilities are used to form the appropriate optimal network topology along with reducing the power consumption. As far as routing the message regarding data is concerned, multihop technologies provide this facility with the use of the networks comprising wireless sensors using internet to a gateway from a IoT node. All in doing this a technology is used known as ZigBee technology in which collected data is ultimately allowed to be accessed via a web server from the internet of the things gateway [13].

To overcome the challenge to communicate with the remote server/devices using internet, the IoT networks protocols are used as a latest advances through the messaging optimized at application level to mitigate the data rates. In such scenario the message queuing telemetry transport (MQTT) protocol is used to gather data through sensors installed at the crop field having a purpose to monitor the greenhouses [14]. Further, at the cloud level for the gathering and analysis of the data from the CPS, these IoT protocols are used. The concept of the FIWARE core comprising a set of auxiliary enablers are employed to approach IoT gateways using a couple of protocols like MQTT/CoAP in order to analyze the historical and current data [15]. Later on real system limiting the technologies and functionalities was proposed. Here, the system used a protocol based on the web over the GPRS along with monitoring characteristics only [16]. Moreover, the IoT protocols in addition to technologies are comprised to real hydroponic system with a weak flexibility in the implementation of the edge computing [17].

Ultimately, a real proposed hydroponic crop management system comprising latest IoT technologies as well as protocols powered by FIWARE dealing with necessary PAg atomic tasks to get performed at the local CPS level orchestrated by virtualized nodes in the control module [3].

In addition to the advances in IoT technologies, the proposed system comprises the generative adversarial nets (GAN). Restricted Boltzmann machine(RBM) [18,21] falls under undirected graphical models rely on the latent variable. Further, these models computing the product of the functions which termed as denormalized and which normalized considering all possible state of random variables using a global integration. However, a plenty of trivial instances of the partition 
functions along with its gradient are computed through Markov Chain Monte Carlo (MCMC) methods $[19,23]$. In this line other undirected graphical models rely on the latent variable are termed as Deep Boltzmann Machine (DBM) [20] including a couple of variants. All the above are nothing but just the options to digraphical models. A module that combines these directed and undirected models together is knows as Deep Belief Networks (DBNs) [21] with specification having a singleton set of undirected layer supported by the set of many directed layers but these modules cope to computational problems even with having approximate layer-wise training criterion.

The Noise-Contrastive Estimation (NCE) [22] used log likelihood supported by the criteria which do not bind or approximate it. The score matching [18] is also included in the same line of the story. NCE uses a discriminative criterion to get trained in order to make the use of generative model. Probability density is used for the learning purpose. Notwithstanding that generated date is discriminated by the generative model from samples a fixed noise distribution leads to a slow learning having learned the model approximately accurate distribution over a bitty subset comprising reviewed data.

Further, we have some methods to train a generative model fetching samples from the required distribution instead of explicitly defining probability distribution which leads to the approach that such model needs to get trained using backpropagation like framework of generative stochastic network (GSN) [23] and uses Markov chain. For sampling purpose the proposed GAN does not need a Markov chain such as to GSN. The main reason is that GANs do not need feedback loops while generating data as they are capable to command piecewise linear units $[29,24,28]$ in order to ameliorate the backpropagation performance having difficulties with unbound activation while employed with ina feedback loop. The latest examples of using backpropagation to train a generative model includes auto-encoding variational Bayes [25] and in the same line stochastic backpropagation work [26]. [1]

\section{System Architecture}

In order to get aware of a system, the understanding of the architecture of the system is very important aspect. The proposed system architecture is exhibited in the fig-1. The entire system is 
bifurcated into three segments and termed as three their system. These three segments are named as local CPS layer installed at the crop side, edge computing layer [3] and the cloud computing - gan layer. CPS layer is responsible to interact with greenhouses field side whereas the edge computing layer also known as intermediate layer in between the system consists of the control modules which virtualized using NFV hunch. Last but not the least plane is called Cloud Computing-GAN plane. This part of the system is basically itself distributed between two parts i.e one is Cloud Computing part and another is termed as GAN (generative adversarial nets). With the help of internet, NFV nodes provide a network route from the crop site to the cloud layer.

In the PAg the local deployed CPS are linked with sensors a long with actuators generally using RS485 industrial serial to gather the information needed for analysis and to manage other parameters such as irrigation, environmental factors etc. respectively. As far as wireless communication is concerned, 6LowPAN is employed for connecting purpose and even CPS units with several technologies (DSL, microwave, fiber optical etc.) using an access network over the internet are interconnected. Atomic level tasks like closing window when needed, managing irrigation time period situationally, ventilating before pre-marked thresh hold violated, alerting unusual electricity cut etc. are performed at the CPS level.

The second layer of the architecture contains the control modules are liable to managing the CPS layer. These control modules are potentially strengthened by NFV. They are monitoring and controlling the nutrition tasks, irrigation tasks, climate and auxiliary tasks. Alerting and power management activities are also managed. This layer controls the planning of gathering the data from the CPS and to provide the same data to the cloud in order to perform the required analysis. The analyzed data is further pushed to the GAN system accordingly for better result due to probabilistic approach with backpropagation training method to enable system functions providing more appropriate decision. This layer provides the path from crop field to the cloud layer by NFV hunch using communication IoT protocol MQTT as it is more usable for the industrial process management. CoAP is also considered to specially assist non-critical tasks without control. This layer provides a fiware based edge solution. 
The third layer comprising two components as cloud computing section as well as GAN section. This platform, through the concept of IoT protocols provided by the edge solution to communicate and gather data from local crop side layer, is used to perform the analysis of the past and current data. The second component of the platform, GAN, is used to ameliorate the system further avoiding the Markov chain method in training the model as it uses backpropagation. This layer also works as an interface to the users.

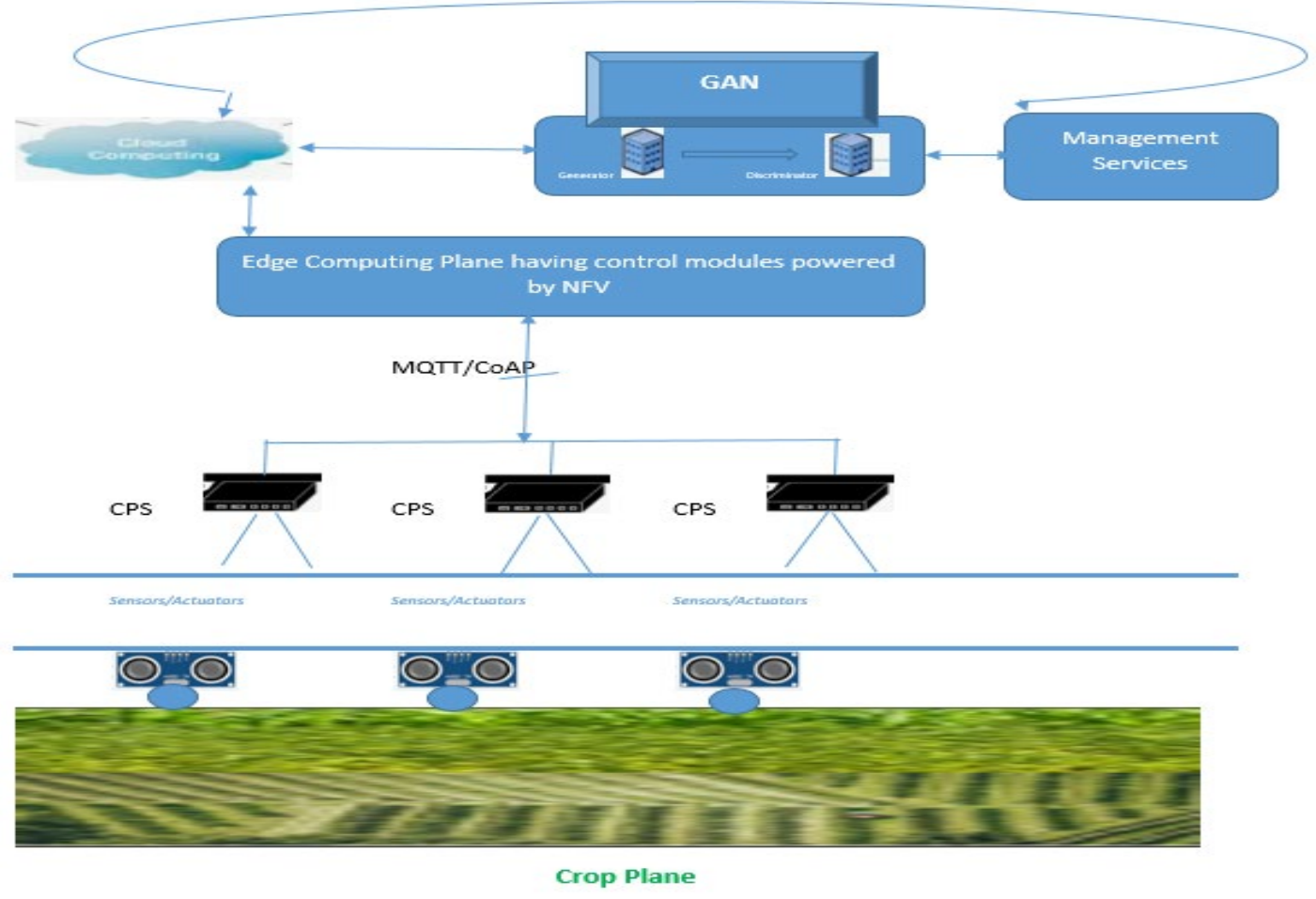

Fig 1. The proposed smart crop system using GAN

In order to send or receive data update notification when a change is made to the maintained data, NGSI interface is employed. 
If the multilayer perceptron generative model is used to generate the needed samples by transporting random noise and in addition the discriminative model also uses multilayer perceptron, then the system in particular context is referred as adversarial nets. Here, the two models are trained by backpropagation algorithms. These models also get trained by dropout algorithm [27]. Due to backpropagation, no Markov chain including approximate inference required. This system works on the concept of the following min max equation as follows:

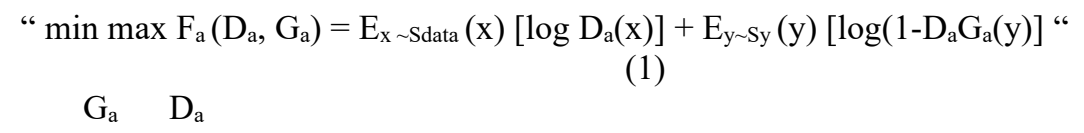

Here, function $F_{a}$ is a value function of $G_{a}$ and $D_{a}$.

$\mathrm{G}_{\mathrm{a}}$ describes a mapping $\mathrm{G}_{\mathrm{a}}\left(\mathrm{y}, \Theta_{\mathrm{g}}\right)$ after defining a noise variable $\mathrm{s}_{\mathrm{y}}(\mathrm{y})$ is a differentiable function with parameter $\Theta_{g}$, for multilayer perceptron. The function $D_{a}$ in equation (1) also represents a mapping for second multilayer perceptron over data $x$ as $D_{a}\left(x, \Theta_{d}\right)$ to produce a scalar quantity where $\Theta_{d}$ is a parameter. $D_{a}(x)$ determines the probability whether $x$ taken from the data instead of generator distribution $\mathrm{s}_{\mathrm{g}}$. The function $\mathrm{D}_{\mathrm{a}}$ is trained to maximize the assignment probability of accurate level to coaching illustrations as well as samples from the function $G_{a}$. Further, $G_{a}$ is also trained in order to mitigate the function $\log \left(1-\mathrm{D}_{\mathrm{a}}\left(\mathrm{G}_{\mathrm{a}}(\mathrm{y})\right)\right)$. [1]

A theoretically working of the system is described further. Once the required data at the cloud part gets analyzed, it is further forwarded to GAN for more filtering to provide more optimized data needs to be used to enhance system performance in order to assist in more production. Generator will generate the data distribution from the data gathered and analyzed at the cloud part and discriminator will discriminate the data in order select more optimized values of the concerned parameters to let the system know when to take more appropriate action to increase system working efficiency to make the optimum use of irrigation, nutrient solution, re-circulation process, climate change and other auxiliary actions.

\section{Merits and Demerits}


Some advantages sighted through GAN are mentioned here. It holds the primary computational advantages using statistical merits from generator due to the probabilistic approach but the change in the dependent variable with respect to the independent variable is used passing to discriminator only. This leads to the fact that generator parameters do not copy the input parts directly. The adversarial nets provide more optimized value to the sharp output due to the presence of the multilayer perceptron discriminator which started to discriminate the required data until discriminator is not able to improve. It means it becomes hard for the discriminator to distinguish the two distribution i.e. $D_{a}(x)=\frac{1}{2}$. Adversarial nets can degenerate the distribution whereas Markov chains need the distribution a bit blurry so that chains to have ability to mix between mode. Markov chain is not needed due to backpropagation. [1]

With many advantages the system has some disadvantages in terms of the use of adversarial nets as well. The hot demerit is that it is not easy to train the GAN as two multilayer perceptron models work to cope each other, ultimately aiming to attain equilibrium i.e. $\mathrm{s}_{\mathrm{g}}=\mathrm{s}_{\text {data. }}$. Notwithstanding without having assurance that convergence would be resulted by competing gradient. After the instability in training the models, mode collapse is another demerit of the system. This situation arises if the generator gets trained to produce the samples only set a somewhat portion of the real distribution i.e. for an adversarial nets getting trained over 5 Gaussian synthetic dataset, the generator can get only trained to set one Gaussian cluster in one time instead of setting all 5 clusters in one go. Another demerit comes with weak gradient. If the difference is large between the real and counterfeit distribution, it is hard to train the system as the discriminator provides weaker gradient signal. Here, in order to get gradient only backpropagation is used.

\section{Conclusion}

The proposed system discusses a three tier system comprising the IoT technologies benefits through automation using communication to transport the updated information for analysis purpose using edge and could computing along with the concept of the generative adversarial nets (GAN) in the field of agronomy termed as precision agriculture in this scenario. Aiming to deploy the system the local layer linked to the crop field in the greenhouses, edge layer monitor and manage the control 
modules through NFV. The final Cloud-GAN layer provides an efficient level of analysis and management using management services along with employing two multilayer perceptron models to assist more accurate and efficient system decision to enhance the crop quality and productivity by controlling and handling effective factors such as irrigation, the optimal use of nutrient solution, climate affecting factors etc.

The proposed system without the use of GAN has already been successfully implemented in the region of south east Spain where greenhouses need to cope with Mediterranean water. That system was implemented on the two phases of the tomato crop (first phase October 2016 to May 2017 \& second phase June 2017 to November 2017) resulting the saving of the water around $30 \%$ and $80 \%$ savings in terms of the nutrient solution which must be used as a re-circulation process to enhance the crops productivity optimizing the costs. [3]

Our theoretically proposed system with the inclusion of adversarial nets will simply assist to enhance the system performance on the certain concerned parameters accordingly due to its generative and discriminative probabilistic approach. As a future work, the actual performance of our proposed system would be judged on the simulation basis by considering more effective parameters to enhance advances in the field of the precision agriculture.

\section{References}

[1]. Ian J. Goodfellow, Jean Pouget-Abadie, Mehdi Mirza, Bing Xu, David Warde-Farley, Sherjil Ozair, Aaron Courville, Yoshua Bengio (2014). Generative Adversarial Nets.

[2]. Resh, H. M. (2012). Hydroponic food production: A definitive guidebook for the advanced home gardener and the commercial hydroponic grower (7th ed.). CRC press.

[3]. Miguel A. Zamora-Izquierdo, Jose Santa, Juan A. Martinez, Vincete Martinez and Antonio F. Skarmeta (2018). Smart farming IoT platform based on edge and cloud computing. Special Issue: Intelligent Systems for Environmental Applications Research Paper. https://doi.org/10.1016/j.biosystemseng.2018.10.014.

[4]. Mulla, D. J. (2013). Twenty-five years of remote sensing in precision agriculture: Key advances and remaining knowledge gaps. Biosystems Engineering, 114, 358-371. Special Issue: Senisng Technologies for Sustainable Agriculture. https:doi.org/10.1016/j.biosystemseng.2012.08.009.

[5]. Atzori, L., Iera, A., \& Morabito, G. (2010). The internet of things: A survey. Computer Networks, 54, 2787-2805. https://doi.org/10.1016/j.comnet.2010.5.010. 
[6]. Ray, P. P. (2017). Internet of things for smart agriculture: Technologies, practices and future direction. Journal of Ambient Intelligence and Smart Environment, 9, 395-420.

[7]. Gebbers, R., and Adamchuk, V. I. (2010). Precesion agriculture and food security. Science, 327, 828-831. https//doi.org/10.1126/science.1183899.

[8]. Riquelme, J. L., Soto, F., Suardiaz, J., Sanchez, P., Iborra, A.and Verra, J., (2009). Wireless sensor networks for precision horticulture in southern Spain. COmputer and Electronics in Agriculture, 68, 25-[35]. $\quad$ https://doi.org/10.1016/j.compag.2009.04.006.

[9]. An, W., Wu, D., Ci, S., Luo, H., Adamchuk, V., and Xu, Z. (2016). Agriculture cyberphysical systems. In Cyber-physical systems: Foundations, principles and applications (pp. 399417). Elsevier. https//doi.org/10.1016/B978-0-12-803801-7.00025-0.

[10]. Canadas, J., Sanchez-Molina, J. A., Rodriguez, F., \& del Aguila, I. M. (2017). Improving automatic climate control with decision support techniques to minimize disease effects in greenhouse tomatoes. Processing in Agriculture, 4, 50-63. https://doi.org/10.1016/j.inpa.2016.12.002.

[11]. Pawlowski, A., Sanchez-Molina, J., Guzman, J., Rodriguez, F. and Dormido, S. (2017). Evaluation of event-based irrigation system control scheme for tomato crops in greenhouses. Agriculture Water Management, 183, 16-25. https://doi.org/10.1016/j.agwat.2016.08.008. Special Issue: Advances on ICTs for Water Management in Agriculture.

[12]. Marhaenanto, B., Soni, P., and Salokhe, V. M. (2013). Development of an internet-based greenhouse control system. International Agriculture Engineering Journal, 22, 72-83.

[13]. Lamprinos, I., Charalambides, M., (2015). Experimental assessment of zigbee as the communication technology of a wireless sensor network for greenhouse monitoring. International Journal of Advanced Smart Sensor Network Systems, 5, 1-10.

[14]. Muthupavithran, S., Akash, S., and Ranjithkumar, P. (2016). Greenhouse monitoring using internet of things. International Journal of Innovative Research in Computer Science and Engineering, 2, 13-19.

[15]. Martinex, R., Paastor, J. A., Alvarez, B., and Iborra, A., (2016). A testbed to evaluate the fiware-based iot platform iin the domain of precision agriculture. Sensors, 16. https://doi.org/10.3390/s16111979.

[16]. Lopez-Riquelme, J., Pavon-Pulido, N., Navarro-Hellin, H., Soto-Valles, F., and TorresSanchez, R., (2017). A software architecture based on fiware cloud for precision agriculture. Agriculture Water Management, 183, 123-135. https://doi.org/10.1016/j.agwat.2016.10.020. Special Issue: Adavances on ICTs for Water Management in Agriculture.

[17]. Ferrandez-Pastor, F. J., Garca-Chamizo, J. M., Neito-Hadalgo, M., Mora-Pascual, J., \& Mora-Martnez, J. (2016). Developing ubiquitous sensor network plateform using internet of things: Application in precision agriculture. Sensors, 16. https://doi.org/10.3390/s16071141. [18]. Smolensky, P. (1986). Information processing in dynamical systems: Foundations of harmony theory. In D. E. Rumelhart and J. L. McClelland, editors, Parallel Distributed Processing, volume 1, chapter 6, pages 194-281. MIT Press, Cambridge.

[19]. Bengio, Y., Mesnil, G., Dauphin, Y., and Rifai, S. (2013a). Better mixing via deep representations. In ICML'13.

[20]. Salakhutdinov, R. and Hinton, G. E. (2009). Deep Boltzmann machines. In AISTATS'2009, pages 448- 455 . 
[21]. Hinton, G. E., Osindero, S., and Teh, Y. (2006). A fast learning algorithm for deep belief nets. Neural Computation, 18, 1527-1554.

[22]. Gutmann, M. and Hyvarinen, A. (2010). Noise-contrastive estimation: A new estimation principle for unnormalized statistical models. In AISTATS'2010.

[23]. Bengio, Y., Thibodeau-Laufer, E., and Yosinski, J. (2014a). Deep generative stochastic networks trainable by backprop. In ICML'14.

[24]. Glorot, X., Bordes, A., and Bengio, Y. (2011). Deep sparse rectifier neural networks. In AISTATS'2011.

[25]. Kingma, D. P. and Welling, M. (2014). Auto-encoding variational bayes. In Proceedings of the International Conference on Learning Representations (ICLR).

[26]. Rezende, D. J., Mohamed, S., and Wierstra, D. (2014). Stochastic backpropagation and approximate inference in deep generative models. Technical report, arXiv:1401.4082.

[27]. Hinton, G. E., Srivastava, N., Krizhevsky, A., Sutskever, I., and Salakhutdinov, R. (2012b). Improving neural networks by preventing co-adaptation of feature detectors. Technical report, arXiv:1207.0580.

[28]. Goodfellow, I. J., Warde-Farley, D., Mirza, M., Courville, A., and Bengio, Y. (2013a). Maxout networks. In ICML'2013.

[29]. Jarrett, K., Kavukcuoglu, K., Ranzato, M., and LeCun, Y. (2009). What is the best multistage architecture for object recognition? In Proc. International Conference on Computer Vision (ICCV’09), pages2146-2153. IEEE. 